\title{
NÚMERO TOTAL DE OPERACIONES DE PATOLOGÍA DIGESTIVA ALTA EN CHILE AÑO 2011*
}

\author{
Dr. Attila Csendes J. ${ }^{1}$ \\ 1 Departamento de Cirugía Hospital Clínico Universidad de Chile. \\ Santiago, Chile.
}

\begin{abstract}
Number of gastrointestinal surgical interventions performed in Chile during 2011

Background: There is paucity of information about the number of gastrointestinal surgical interventions in Chile. Aim: To report the number of gastrointestinal operations carried out in Chile during 2011. Material and Methods: Review of databases from the Department of Statistics and Health care information of the Ministry of Health. The two most common gastrointestinal surgical interventions and four highly complex interventions were selected. Results: The most common gastrointestinal intervention was cholecystectomy in 52.000 cases, followed by appendectomy in 37.000 cases. A gastrectomy for gastric cancer was performed in 1.032 cases, performing a total gastrectomy in $74 \%$ and with a $2.9 \%$ mortality. Esophagectomy was carried out in 42 patients with an overall mortality of $9.5 \%$. A pancreatectomy for pancreatic cancer was performed in 102 patients (pancreatoduodenectomy in $68 \%$ ), with an overall mortality of $5.9 \%$. Bariatric surgery was performed in 2.834 cases (vertical gastrectomy in 70\% and gastric bypass in 30\%). Conclusions: Cholecystectomy was by far the most common surgical procedure performed. Esophagectomy and pancreatectomy were associated with a high mortality rate. Gastrectomy for gastric cancer had a low postoperative mortality.

Key words: Cholecystectomy, gastrectomy, pancreatectomy.
\end{abstract}

\section{Resumen}

Introducción: No hay datos oficiales sobre el número total de intervenciones quirúrgicas referentes a patología gastrointestinal alta. Objetivo: Conocer algunos datos estadísticos al respecto. Material y Método: Se obtuvo del Departamento de Estadísticas e Informaciones de Salud del Ministerio de Salud el total de intervenciones realizadas el año 2011 en Chile, seleccionando las 2 operaciones más frecuentes y 4 operaciones de alta complejidad definidas por la patología de base, la complejidad del acto quirúrgico y la mortalidad operatoria. Resultados: Después de la cesárea, la colecistectomía es la operación más frecuente (52.000 pacientes) seguida de la apendicectomía (37.000 pacientes). La gastrectomía por cáncer gástrico se realizó en 1.032 pacientes con una mortalidad operatoria global de 2,9\%. La gastrectomía total se efectuó en $74 \%$ de los casos. La esofaguectomía se realizó en 42 pacientes con mortalidad global de 9,5\%. La pancreatectomía por cáncer de páncreas se efectuó en 102 pacientes con una mortalidad global de 5,9\%, siendo la

*Recibido el 13 de mayo de 2014 y aceptado para publicación el 22 de junio de 2014.

El autor no refiere conflictos de interés.

Correspondencia: Dr. Attila Csendes J. acsendes@hcuch.cl 
pancreatoduodenectomía la intervención más frecuente (68\%). La cirugía bariátrica comprende 2 operaciones con un total de 2.834 casos, con una proporción de $70 \%$ para la gastrectomía vertical y un $30 \%$ para el bypass gástrico. Conclusiones: La colecistectomía es la intervención quirúrgica no obstétrica más frecuente. La esofaguectomía y la pancreatectomía, se realizan en un bajo número de pacientes, siendo la mortalidad operatoria de ambos procedimientos alta. La gastrectomía por cáncer gástrico tiene una baja mortalidad operatoria. Se reporta por primera vez una cifra oficial referente al número de cirugías bariátricas.

Palabras clave: Colecistectomía, pancreatectomía, esofaguectomía, gastrectomía por cáncer gástrico.

\section{Introducción}

El año 2008 publicamos un estudio respecto a las intervenciones quirúrgicas realizadas en Chile los años 2004 y 2005, incluyendo sólo procedimientos sobre el tracto gastrointestinal alto, excluyendo patología colo-rectal ${ }^{1}$. En esa época no había datos suficientes y confiables respecto al área privada de salud. El objetivo del presente estudio fue determinar el total de operaciones realizadas en el país, en ambos sistemas de salud, con especial énfasis a 4 operaciones de alta complejidad, incluyendo por primera vez una estadística oficial sobre el número de operaciones bariátricas realizadas.

\section{Material y Método}

Con el apoyo y ayuda del Departamento de Estadísticas e Información de Salud (DEIS) del Ministerio de Salud, se nos proporcionó el número total de intervenciones realizadas el año 2011 en Chile, tanto del área pública como privada. Se comparan con los resultados obtenidos en la publicación anterior, que es un promedio anual entre los años 2004 y 2005. También se analizó la mortalidad operatoria de 3 operaciones de alta complejidad.

\section{Resultados}

En la Tabla 1 se muestran las operaciones más frecuentes de cirugía abdominal comparadas con las cesáreas realizadas en Chile durante el año 2011. La colecistectomía se realizó en un poco más de 50.000 pacientes mientras que la apendicectomía por apen- dicitis aguda se ubicó en el $2^{\circ}$ lugar. Con respecto a la patología biliar, se aprecia lo siguiente:

a. Hay un aumento de 20.000 colecistectomías comparado con el promedio anual de los años 2004-2205.

b. La colecistectomía laparoscópica se efectúa en el país en $82 \%$ de los pacientes.

c. Hay aún un $15 \%$ de las operaciones que se realizan por vía laparotómica.

d. La coledocostomía sólo se practicó en un 3,3\% del total de colecistectomías.

En la Tabla 2 se señalan 3 operaciones que se definen como de alta complejidad, tanto por la patología tumoral de base como por la complejidad del acto quirúrgico, la elevada morbilidad postoperatoria y la alta mortalidad operatoria reconocida internacionalmente.

En la publicación anterior no existían datos referentes a la mortalidad operatoria. Al analizar a los pacientes con cáncer gástrico sometidos a gastrectomía, se observa que:

a. La gastrectomía subtotal se efectuó en el $23 \%$ de los pacientes con una mortalidad operatoria de $2,9 \%$.

b. La gastrectomía proximal es una operación excepcional, ya que se realizó en 3\% de los casos sin mortalidad.

c. La gastrectomía total es la operación de elección en Chile para estos pacientes, practicándola en el $74 \%$ de los casos, con una mortalidad nacional de $3,4 \%$.

La esofaguectomía se efectuó en 42 pacientes con cáncer de esófago, con una mortalidad operatoria de

Tabla 1. Operaciones más frecuentes en Chile

\begin{tabular}{|lccc|}
\hline Operación & Promedio 2004-2005 & n de pacientes & 2011 \\
1. Cesárea & - & 62.224 & \% aumento \\
2. Colecistectomía & 29.850 & 51.877 & \\
- Laparoscópica & - & 82.483 & $(81,9 \%)$ \\
- Laparotómica & - & $7.669(14,8 \%)$ & \\
- Coledocostomía & - & $1.725 \quad(3,3 \%)$ & 19,3 \\
\hline 3. Apendicectomía & 30.997 & 36.993 & \\
\hline
\end{tabular}


Tabla 2. Operaciones de alta complejidad en Chile

\begin{tabular}{|c|c|c|c|c|}
\hline \multirow[t]{2}{*}{ Operación } & \multicolumn{2}{|c|}{ Promedio } & \multirow[t]{2}{*}{$\%$ del total } & \multirow[t]{2}{*}{ Mortalidad } \\
\hline & 2004-2005 & 2011 & & \\
\hline 1. Gastrectomía por cáncer gástrico & 710 & 1.032 & & $33(3,2 \%)$ \\
\hline Subtotal & - & 237 & 23 & $7(2,9 \%)$ \\
\hline Normal & - & 31 & 3 & 0 \\
\hline Total & - & 764 & 74 & $26(3,4 \%)$ \\
\hline 2. Esofaguectomía por Cáncer esófago & 63 & 42 & & $4(9,5 \%)$ \\
\hline 3. Pancreatectomía por Cáncer páncreas & 90 & 102 & & $6(5,9 \%)$ \\
\hline Parcial & - & 26 & 25,5 & - \\
\hline Total & - & 7 & 6,8 & - \\
\hline Pancreatoduodenectomía & - & 69 & 67,7 & $6(8,7 \%)$ \\
\hline
\end{tabular}

9,5\%. La pancreatectomía por cáncer de páncreas se realizó en 102 pacientes con una mortalidad operatoria de 5,9\%. Esta mortalidad ocurrió exclusivamente en pacientes sometidos a una pancreatoduodenectomía, con una cifra de $8,7 \%$ mientras que la pancreatectomía parcial o total, que se realizó en cerca de $32 \%$ de los pacientes, no registró mortalidad.

La Tabla 3 señala el total de cirugías bariátricas realizadas, correspondiendo en 70,8\% a la gastrectomía vertical y en 29,2\% al bypass gástrico. No se obtuvo la cifra de mortalidad operatoria.

\section{Discusión}

Los resultados del presente estudio muestran que el año 2011 se realizaron algo más de 50.000 colecistectomías, con claro aumento en relación a los años 2004 y 2005, siendo la operación más frecuentemente realizada en Chile después de las cesáreas, que se realizaron en 62.000 pacientes. En segundo lugar, se observa que hay 2 operaciones de alta complejidad, como son la esofaguectomía y la pancreatoduodenectomía que tienen una elevada mortalidad operatoria cercana al 9\%. En tercer lugar, el número de estas operaciones es muy bajo en Chile, para una población cercana a 17 millones de personas. En cuarto lugar, la gastrectomía por cáncer gástrico se realizó en algo más de 1.000 pacientes con una mortalidad operatoria general de 3\%. Por último, es la primera vez que se reportan cifras oficiales anuales de cirugía bariátrica en Chile y la proporción de los 2 únicos procedimientos que se realizan en nuestro país.

Un punto muy importante que señalar se refiere al hecho que la previa publicación nuestra que reportaba los datos de los años 2004 y 2005 sólo incluía datos completos de las operaciones realizadas por el Ministerio de Salud, ya que no se logró obtener
Tabla 3. Cirugía de obesidad mórbida. Año 2011

\begin{tabular}{|lrl|}
\hline - Gastrectomía vertical & 2.006 & $(70,7 \%)$ \\
- Bypass gástrico & 828 & $(29,2)$ \\
- Total & 2.834 & \\
\hline
\end{tabular}

cifras confiables y completas del sector privado. Sin embargo, en las estadísticas del año 2011 se incluyen tanto los datos del sistema público y privado y, por lo tanto, representa la verdadera realidad nacional. Este hecho explica el primer punto que se refiere a esta discrepancia en más de 20.000 pacientes que se sometieron a colecistectomía. Si asumimos que el sistema público operó a 30.000 pacientes de colecistectomía (60\%) se aprecia que casi un $40 \%$ se atendió en el sistema privado, lo que demuestra que ambos sistemas (público y privado) logran un fuerte impacto en la solución de este problema. Comentario similar se aplica a las apendicectomía con un aumento de $19 \%$.

Un hecho similar ocurre con las gastrectomías por cáncer gástrico, en que entre los 2 períodos de tiempo analizados, aumentó en casi $30 \%$. Esto por una parte se podría explicar por el impacto del plan AUGE en una conducta quirúrgica más precoz y por un mayor acceso de estos pacientes a una operación de alto costo. Pero también es posible postular que un porcentaje de estos pacientes $(30 \%)$ también se han operado en el sistema privado, cuyas cifras no estaban claramente establecidas en el estudio previo. La cifra de mortalidad operatoria nacional de $3 \%$ en gastrectomía por cáncer gástrico revela un manejo médico multidisciplinario muy adecuado, probablemente influenciado por las guías clínicas del Plan AUGE y, por lo tanto, el cumplimiento obligatorio de los plazos estipulados para atender a estos pa- 
cientes. Por otra parte, demuestra que la dedicación muy interesada de numerosos grupos quirúrgicos a lo largo de Chile por varias décadas a esta patología, ha permitido reducir sustancialmente la mortalidad operatoria, que en 1970 era de $30 \%$ a una cifra de $3 \%$, es decir, 10 veces menor ${ }^{2,3}$.

Otro hecho importante del presente estudio se refiere a dos operaciones de alta complejidad, cuyas cifras de mortalidad global en el país no eran conocidas. Es frecuente observar el hecho que los reportes de estas operaciones vienen de centros quirúrgicos especializados, que reportan cifras de morbilidad y mortalidad muy aceptables, similares a la de centros internacionales, pero que no son reproducibles ni muestran la verdadera realidad nacional, por lo que son sólo aplicables a esos escasos centros especializados. En estas dos operaciones de alta complejidad, la esofaguectomía y la pancreatectomía destacan 2 hechos importantes para la Salud Pública Chilena: por una parte es llamativo el escaso número de pacientes operados durante 1 año en todo Chile $y$, por otra parte, llama la atención la elevada cifra de mortalidad operatoria: En nuestro Hospital Clínico Universitario, las cifras registradas por nuestra Unidad de Oncología durante los últimos 8 años muestran una mortalidad operatoria de $3 \%$ para la pancreatectomía y de 4,5\% para la Esofaguectomía. Estas cifras refuerzan una vez más el hecho que hemos insistido tantas veces en diferentes ocasiones: el Ministerio de Salud debe designar 2 ó 3 centros quirúrgicos que sean capaces de concentrar estas patologías, para así, por el alto volumen anual de operaciones, poder obtener una cifra de mortalidad operatoria menor al 4\%. Mientras no se concrete esta decisión, muchos centros seguirán operando 1 ó 2 pacientes por año, no logrando obtener una curva de aprendizaje ni el volumen necesario para disminuir dramáticamente esta mortalidad. Los grupos quirúrgicos por su estructura intrínseca, difícilmente trasladan sus pacientes a otros centros, ya que sienten que son capaces de resolver estos problemas.

Finalmente, un logro destacable de este estudio fue obtener por primera vez una cifra oficial nacional referente a cirugía bariátrica. El año 2011 se rea- lizaron cerca de 3.000 operaciones, probablemente la inmensa mayoría en el sector privado ya que estas técnicas no han sido desarrolladas ampliamente en el sector público. Lamentablemente no tenemos los datos de mortalidad operatoria de estos pacientes. Sin embargo, destaca el hecho de la masificación de la gastrectomía vertical laparoscópica (llamada comúnmente manga gástrica) por sobre el bypass gástrico, que es el gold standard de la cirugía bariátrica.

Este hecho se explica en parte porque la gastrectomía vertical parece ser a primera vista una operación "más fácil" que el bypass gástrico. Pero, por otra parte, se podría explicar por el aumento de esta operación en casos con un IMC bajo $35 \mathrm{~kg} / \mathrm{m}^{2}$, que es el estándar científico internacional para indicar la cirugía en estos pacientes. Hay varios grupos quirúrgicos que están realizando esta operación con IMC de 32 ó 33 por motivos más bien estéticos que médicos y esto es una llamada de alerta para una evaluación futura más cuidadosa y profunda sobre este tema.

\section{Agradecimientos}

Agradecemos profundamente la gentil colaboración de la Sra. Ana Valeska Ortiz Labbé y de la Sra. Marcela Soto Aguilera, del Departamento de Estadísticas e Información de Salud (DEIS) del Ministerio de Salud por la información proporcionada referente a las operaciones en Chile.

\section{Referencias}

1. Csendes A, González G. Cirugías digestivas más frecuentes en Chile excluyendo colorrectal. Rev Chil Cir. 2008;60:379-86.

2. Otaiza E, Lopetegui G, Csendes A. Operabilidad y resecabilidad del cáncer gástrico. Rev Med Valpo. 1969;22:229-32.

3. Csendes A, Díaz H, Santa Cruz V, Flores N. Resultados inmediatos de la cirugía en el cáncer gastroesofágico. Arch Soc Cir Chile 1972;24:142-6. 